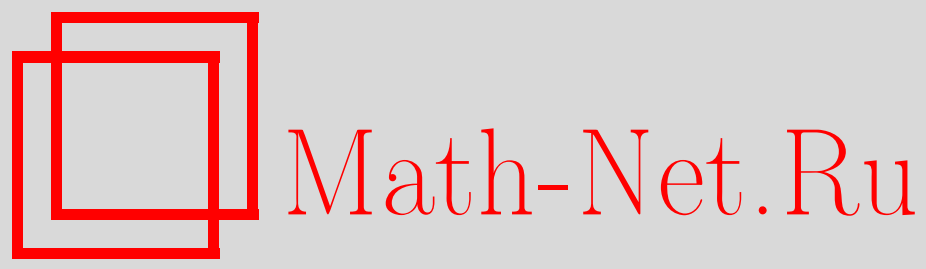

T. Schreiber, Limit theorems for certain functionals of unions of random closed sets, Теория вероятн. и ее примен., 2002, том 47, выпуск 1, 130-142

DOI: https://doi.org/10.4213/tvp3004

Использование Общероссийского математического портала MathNet.Ru подразумевает, что вы прочитали и согласны с пользовательским соглашением

http://www . mathnet.ru/rus/agreement

Параметры загрузки:

IP : 3.80 .181 .102

26 апреля 2023 г., 17:54:05

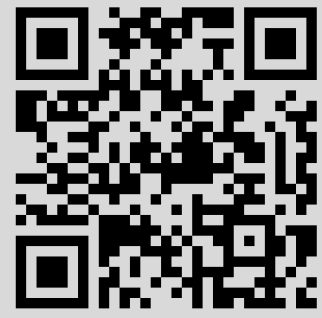




\section{LIMIT THEOREMS FOR CERTAIN FUNCTIONALS OF UNIONS OF RANDOM CLOSED SETS}

Пусть $X_{1}, X_{2}, \ldots$ - последовательность случайных независимых, одинаково распределенных, замкнутых подмножеств некоторого локально компактного хаусдорфова сепарабельного пространства $E$. Для каждого случайного замкнутого множества $Y$ мы рассматриваем функционал $Q_{Y}(F)$, равный вероятности того, что $Y$ не пересекается с замкнутым подмножеством $F \subseteq E$. Цель статьи - установить предельные теоремы для случайных величин $Q_{Y}\left(X_{1} \cup \cdots \cup X_{n}\right)$. Полученные результаты применяются для асимптотического анализа средней ширины выпуклых оболочек, порожденных равномерными выборками на многомерном шаре.

Ключевые слова и фразы: случайные множества, объединения случайных множеств, функционалы достижения, экстремальные значения, выпуклые оболочки, средняя ширина, периметр.

1. Introduction and main results. Consider a locally compact, Hausdorff and separable topological space $E$ and denote by $\mathscr{F}:=\mathscr{F}(E)$ the family of all its closed subsets. We endow $\mathscr{F}$ with the vague (Fell) topology $\mathscr{T}_{f}$ generated by subfamilies of the form

$\mathscr{F}_{G_{1}, \ldots, G_{n}}^{K}:=\left\{F \in \mathscr{F} \mid F \cap K=\varnothing, F \cap G_{1} \neq \varnothing, \ldots, F \cap G_{n} \neq \varnothing\right\}, \quad n \geqslant 0$,

where $K$ runs through the class of compacts and $G_{1}, \ldots, G_{n}$ are open (see Chapter 1 in [9] or Section 1.1 in [10]). It is known that $\left(\mathscr{F}, \mathscr{T}_{f}\right)$ is Hausdorff, compact and separable (see ibidem).

The random elements taking values in $\left(\mathscr{F}, \mathscr{B}_{f}\right)$, where $\mathscr{B}_{f}$ is the Borel $\sigma$-field corresponding to $\mathscr{T}_{f}$, will be referred to as random closed sets (for extensive references see [6], [9] or [10]). Without a further mention we will assume all the considered random elements to be defined on a common probability space $(\Omega, \Im, \mathbf{P})$.

For a random closed set $Y$ its avoidance functional $Q_{Y}: \mathscr{F} \rightarrow[0,1]$ is defined by $Q_{Y}(F):=\mathbf{P}\{F \cap Y=\varnothing\}$. A number of important functionals on $\mathscr{F}$ can be expressed in terms of $Q_{Y}$ for properly chosen $Y$. For example taking an arbitrary $E$-valued random element $V$ and setting $Y$ to be the

${ }^{*}$ Faculty of Mathematics and Computer Science, Nicholas Copernicus University, Toruń, Poland; e-mail: tomeks@mat.uni.torun.pl 
singleton $\{V\}$ we get $Q_{Y}(F)=\mathbf{P}\{V \notin F\}=1-\mu(F)$, where $\mu$ is the distribution of $V$. Further examples are given in the next section.

Define for $F \in \mathscr{F}$

$$
f(F):=Q_{Y}(F)
$$

let $X, X_{1}, X_{2}, \ldots$ be an i.i.d. sequence of random closed sets jointly independent of $Y$ and denote $X^{(n)}:=X_{1} \cup X_{2} \cup \cdots \cup X_{n}$. The main purpose of this paper is to develop a tool for investigating the asymptotic behaviour of $f\left(X^{(n)}\right)$.

Consider the capacity (hitting) functional of $X$ defined on $\mathscr{F}$ by

$$
T_{X}(F):=\mathbf{P}\{F \cap X \neq \varnothing\}=1-Q_{X}(F)
$$

(see Chapter 2 in [9] or Section 1.2 in [10]). Observe that $T_{X}$ is nondecreasing in that $T_{X}\left(F_{1}\right) \leqslant T_{X}\left(F_{2}\right)$ for $F_{1} \subseteq F_{2}$. Further, $T_{X}$ is Borel measurable because the function $\mathscr{F} \times \mathscr{F} \ni\left(F_{1}, F_{2}\right) \mapsto \mathbf{1}_{\left\{F_{1} \cap F_{2} \neq \varnothing\right\}}$ is Borel measurable (see [9, Corollary 1 on Proposition 1.2.4]) and $T_{X}(F)=\mathbf{E}_{\{X \cap F \neq \varnothing\}}$. In particular, $T_{X}(Y)$ is a well-defined random variable. Denote by $\Psi:=\Psi_{X, Y}$ its distribution function, i.e.,

$$
\Psi(t):=\Psi_{X, Y}(t):=\mathbf{P}\left\{T_{X}(Y) \leqslant t\right\} .
$$

The following theorem gives us a rough description of the expectation $\mathbf{E} f\left(X^{(n)}\right)=\mathbf{P}\left\{X^{(n)} \cap Y=\varnothing\right\}$ for large $n$.

Theorem 1. With the notation introduced above

$$
\begin{aligned}
-\limsup _{t \rightarrow 0} \frac{\ln \Psi(t)}{\ln t} & \leqslant \liminf _{n \rightarrow \infty} \frac{\ln \mathbf{P}\left\{X^{(n)} \cap Y=\varnothing\right\}}{\ln n} \\
& \leqslant \limsup _{n \rightarrow \infty} \frac{\ln \mathbf{P}\left\{X^{(n)} \cap Y=\varnothing\right\}}{\ln n} \leqslant-\liminf _{t \rightarrow 0} \frac{\ln \Psi(t)}{\ln t} .
\end{aligned}
$$

A much stronger theorem can be proven if we know more about the behaviour of $\Psi$ in the neighbourhood of 0 .

Theorem 2. Assume that $\Psi(0)=0$ and suppose that $\Psi$ is absolutely continuous in some neighbourhood of 0 with density $\varphi$ admitting the representation

$$
\varphi(t)=c t^{\alpha}+o\left(t^{\alpha}\right)
$$

where $c$ is a positive constant and $\alpha>-1$. Then

$$
\mathbf{E} f\left(X^{(n)}\right)=\mathbf{P}\left\{X^{(n)} \cap Y=\varnothing\right\}=c \Gamma(\alpha+1) n^{-(\alpha+1)}+o\left(n^{-(\alpha+1)}\right) .
$$

Note that relation (4) cannot be replaced by weaker assumption that $\varphi$ is regularly varying in 0 with index greater than -1 (see, e.g., Section 0.4 in [14]). To see it take for instance $\varphi(t)=-\ln t$ which is slowly varying at 0 but does not admit the representation (4) and observe, mimicking the proof of Theorem 2, that for such $\varphi$ we have $n \mathbf{E} f\left(X^{(n)}\right) \rightarrow \infty$ and $n^{\beta} \mathbf{E} f\left(X^{(n)}\right) \rightarrow 0$ for $\beta<1$. 
The statements presented so far give us information about the behaviour of $\mathbf{E} f\left(X^{(n)}\right)=\mathbf{P}\left\{X^{(n)} \cap Y=\varnothing\right\}$ only. However, it is easily seen that taking $Y_{1}, \ldots, Y_{k}$ to be i.i.d. copies of $Y$ we have $f^{k}(F)=Q_{Y}^{k}(F)=Q_{Y^{(k)}}(F)$, where $Y^{(k)}:=Y_{1} \cup \cdots \cup Y_{k}$. Assuming that $X_{1}, X_{2}, \ldots, Y_{1}, Y_{2}, \ldots$ are jointly independent we obtain in addition $\mathbf{E} f^{k}\left(X^{(n)}\right)=\mathbf{P}\left\{X^{(n)} \cap Y^{(k)}=\varnothing\right\}$. This remark suggests that we could apply Theorems 1 and 2 for $Y^{(k)}$ instead of $Y$ to obtain analogous results for higher moments of $f\left(X^{(n)}\right)$. The first of these results, extending Theorem 1 , holds under very weak assumptions but is rather rough.

Theorem 3. Assume that there exists (possibly infinite) limit

$$
\gamma=\lim _{t \rightarrow 0} \frac{\ln \Psi(t)}{\ln t} .
$$

Then

$$
\lim _{n \rightarrow \infty} \frac{\ln \mathbf{E} f^{k}\left(X^{(n)}\right)}{\ln n}=-k \gamma
$$

Clearly, relation (5) (for finite $\gamma$ ) is satisfied whenever $\Psi$ is regularly varying at 0 with index $\gamma$. Note however that (5) is, as well, fulfilled for instance by $\Psi(t):=\exp ([\ln t])$ (with $\gamma=1$ ), which is not regularly varying (see Section 0.4 in [14]).

The next statement is a generalisation of Theorem 2. It requires an additional regularity assumption (REG) which says, roughly speaking, that if closed sets $F_{1}, F_{2} \in \mathscr{F}$ are such that the probabilities $T_{X}\left(F_{i}\right)=\mathbf{P}\{X \cap$ $\left.F_{i} \neq \varnothing\right\}, i=1,2$, are small enough then the probability $\mathbf{P}\left\{X \cap F_{1} \neq\right.$ $\left.\varnothing \wedge X \cap F_{2} \neq \varnothing\right\}$ becomes negligibly small. To formulate the condition denote for $F_{1}, F_{2} \in \mathscr{F}$

$$
\Lambda_{X}\left(F_{1}, F_{2}\right):=\mathbf{P}\left\{X \cap F_{1} \neq \varnothing \wedge X \cap F_{2} \neq \varnothing\right\} .
$$

We require that

(REG) There exists $\varepsilon>0$ such that

$$
\mathbf{E}\left(\Lambda_{X}\left(Y_{1}, Y_{2}\right) \mathbf{1}_{\left\{T_{X}\left(Y_{1}\right) \leqslant t\right\}} \mathbf{1}_{\left\{T_{X}\left(Y_{2}\right) \leqslant t\right\}}\right)=O\left(t^{1+\varepsilon} \Psi^{2}(t)\right) .
$$

Then we have the following theorem.

Theorem 4. Under the assumptions of Theorem 2 and condition (REG) we have

$$
\mathbf{E} f^{k}\left(X^{(n)}\right)=(c \Gamma(\alpha+1))^{k} n^{-k(\alpha+1)}+o\left(n^{-k(\alpha+1)}\right) .
$$

Note that in view of Theorem 2 this yields $\mathbf{E} f^{k}\left(X^{(n)}\right)=\left(\mathbf{E} f\left(X^{(n)}\right)\right)^{k}+$ $o\left(n^{-k(\alpha+1)}\right)$. Hence, as a conclusion we get

Corollary 1. With the conditions of Theorem 4

$$
\mathbb{E}\left(f\left(X^{(n)}\right)-\mathbb{E} f\left(X^{(n)}\right)\right)^{k}=o\left(n^{-k(\alpha+1)}\right) .
$$


Finally, we conclude from Corollary 1 that $n^{\alpha+1} f\left(X^{(n)}\right)$ converges in probability to a constant.

Theorem 5. Under the assumptions of Theorem 4

$$
\lim _{n \rightarrow \infty} n^{\alpha+1} f\left(X^{(n)}\right)=c \Gamma(\alpha+1)
$$

in probability.

It is worth noting that while Theorems 1,2 and 3 hold under rather general assumptions, condition (REG) required in Theorems 4 and 5 is much more restrictive. To see a simple example of a situation when (REG) fails consider a sequence $\eta, \xi_{1}, \xi_{2}, \ldots$ of i.i.d. random variables distributed uniformly on $[0,1]$ and take $E:=[0,1], X_{i}:=\left[0, \xi_{i}\right]$ and $Y:=[\eta, 1]$. Clearly then $f\left(X^{(n)}\right)=1-\max _{1 \leqslant i \leqslant n} \xi_{i}$ and a straightforward calculation yields $\Psi(t)=t$ and $\mathbf{E}\left(\Lambda_{X}\left(Y_{1}, Y_{2}\right) \mathbf{1}_{\left\{T_{X}\left(Y_{1}\right) \leqslant t\right\}} \mathbf{1}_{\left\{T_{X}\left(Y_{2}\right) \leqslant t\right\}}\right)=t^{3} / 3$ so that (REG) is not satisfied. On the other hand, an important example of a situation when (REG) holds is given in the next section, where we apply the general results presented above to investigate the asymptotics of the mean width of convex hulls generated by uniform samples on a multidimensional ball.

2. Approximation of a ball by random polytopes. Let $\zeta, \zeta_{1}, \zeta_{2}, \ldots$ be a sequence of i.i.d. random vectors distributed uniformly on the unit ball $B_{d} \subset \mathbf{R}^{d}, d \geqslant 2$, centred in 0 . By $C_{n}$ we denote the convex hull generated by the sample of size $n$, i.e., $C_{n}=\operatorname{conv}\left\{\zeta_{1}, \ldots, \zeta_{n}\right\}$. Recall that for a nonempty compact convex set $K$ its support function (see Section 1.7 in [16]) is defined by $h(K, u):=\max _{x \in K}\langle x, u\rangle, u \in \mathbf{R}^{d}$, where $\langle\cdot, \cdot\rangle$ denotes the scalar product. The main purpose of the current section is to use the results developed so far to prove a limit theorem for the mean width $b\left(C_{n}\right)$ of $C_{n}$ (see (1.7.2) in [16]), given by

$$
b\left(C_{n}\right):=\frac{2}{\omega_{d}} \int_{\mathscr{S}_{d-1}} h\left(C_{n}, u\right) d \sigma(u),
$$

where $\sigma$ denotes the surface measure on the unit sphere $\mathscr{S}_{d-1}:=\partial B_{d}$ and $\omega_{d}:=\sigma\left(\mathscr{S}_{d-1}\right)$.

The asymptotic properties of the convex hulls generated by random samples have been thoroughly investigated in the literature since the pioneering papers by Efron [3] and Rényi \& Sulanke [13]. An extensive survey of these and related results can be found in [15]. In the particular two-dimensional case very strong asymptotic theorems have been obtained during the past decades since Groeneboom's work [4] followed by a number of other authors (see e.g. [1], [2], [5] and [7]). In higher dimensions the results obtained so far are much weaker, nevertheless important progress has been made in investigating the asymptotics of the volume outside the convex hull (see [8] and the references therein). The random processes generated by the support functions of successive convex hulls have been considered in [11]. 
The main result of this section is

Theorem 6. For all $k \in \mathbf{N}$ we have

$$
\mathbf{E}\left(2-b\left(C_{n}\right)\right)^{k}=\left(a_{d}\right)^{k} n^{-2 k /(d+1)}+o\left(n^{-2 k /(d+1)}\right) .
$$

Moreover

$$
\lim _{n \rightarrow \infty} n^{2 /(d+1)}\left(2-b\left(C_{n}\right)\right)=a_{d}
$$

in probability, where

$$
a_{d}:=2 \Gamma\left(\frac{2}{d+1}\right)\left(\frac{\kappa_{d}}{\kappa_{d-1}}\right)^{2 /(d+1)}(d+1)^{-(d-1) /(d+1)}
$$

with $\kappa_{d}$ and $\kappa_{d-1}$ denoting the volumes of $B_{d}$ and $B_{d-1}$, respectively.

It is worth noting that both the convergence rate and the degeneracy of the limiting distribution of $b\left(C_{n}\right)$ have their analogues in the results obtained by Küfer [8] for the volume outside the convex hull $D_{n}:=\operatorname{vol}\left(B_{d} \backslash C_{n}\right)$ (see Theorem 2 therein). Further, in the two-dimensional case Theorem 6 applies to the asymptotic behaviour of the perimeter $L\left(C_{n}\right)$ of $C_{n}$ because for a compact convex set $K \subset \mathbf{R}^{2}$ we have $L(K)=\pi b(K)$ (see p. 210 in [16]). As a particular conclusion we obtain $n^{2 / 3} \mathbf{E}\left(2 \pi-L\left(C_{n}\right)\right) \rightarrow \pi a_{2}$ which agrees with Theorem 7 in [1].

We argue that Theorem 6 is a conclusion from Corollary 1 and Theorem 5 and, hence, the proof of the former reduces to the verification of appropriate conditions assumed in the latter results. To see it observe first that, by the definition of the support function, $h\left(C_{n}, u\right)=\max _{1 \leqslant i \leqslant n} h\left(\left\{\zeta_{i}\right\}, u\right)$, $u \in \mathbf{R}^{d}$. Thus, denoting by hypo $(h(K, \cdot))$ the hypograph of the restriction of the support function of $K$ to the unit sphere $\mathscr{S}_{\boldsymbol{d}-1}$, i.e., hypo $(h(K, \cdot)):=$ $\left\{(u, y) \in \mathscr{S}_{d-1} \times \mathbf{R} \mid y \leqslant h(K, u)\right\}$, we conclude that

$$
\operatorname{hypo}\left(h\left(C_{n}, \cdot\right)\right)=\bigcup_{i=1}^{n} \operatorname{hypo}\left(h\left(\left\{\zeta_{i}\right\}, \cdot\right)\right) \text {. }
$$

To proceed, consider two random elements $\xi$ and $\eta$ distributed uniformly on $\mathscr{S}_{d-1}$ and $[0,1]$ respectively and such that the sequence $\xi, \eta, \zeta, \zeta_{1}, \ldots$ is jointly independent. Observe that $\mathbf{P}\{(\xi, \eta) \notin$ $\operatorname{hypo}(g)\}=1-\left(\omega_{d}\right)^{-1} \int_{\mathscr{S}_{d-1}} \max (0, \min (1, g(u))) d \sigma(u)$ for arbitrary measurable $g: \mathscr{S}_{d-1} \rightarrow \mathbf{R}$. Thus, setting $E:=\mathscr{S}_{d-1} \times[-1,1], \quad X_{i}:=$ $\operatorname{hypo}\left(h\left(\left\{\zeta_{i}\right\}, \cdot\right)\right) \cap E$ (note that $X_{i}$ is closed due to the continuity of $h\left(\left\{\zeta_{i}\right\}, \cdot\right)$ ) and $Y:=\{(\xi, \eta)\}$, according to (1) and (9) we get what follows

$$
f\left(X^{(n)}\right)=Q_{Y}\left(X^{(n)}\right)=1-\frac{1}{\omega_{d}} \int_{\mathscr{S}_{d-1}} \max \left(0, h\left(C_{n}, u\right)\right) d \sigma(u) .
$$

Since the probability that $h\left(C_{n}, \cdot\right)$ admits negative values (which is equivalent to $0 \notin C_{n}$ ) decreases exponentially with $n$ (see the discussion in [8]), so does the probability that the right-hand side of the above identity 
is different from $1-b\left(C_{n}\right) / 2$. Therefore in order to prove Theorem 6 we can replace $2-b\left(C_{n}\right)$ by $2 f\left(X^{(n)}\right)$. The distribution function $\Psi_{X, Y}$ given in (3) has with the current notation the particular form $\Psi_{X, Y}(t)=$ $\left(\omega_{d}\right)^{-1} \int_{\mathscr{S}_{d-1}} \int_{[0,1]} \mathbf{1}_{\{\mathbf{P}\{h(\{\zeta\}, u) \geqslant s\} \leqslant t\}} d s d \sigma(u)$. Hence, defining $c\left(B_{d}, u, s\right)$, $u \in \mathscr{S}_{d-1}, s \in[0,1]$, to be the cap $c\left(B_{d}, u, s\right):=\left\{v \in B_{d} \mid\langle v, u\rangle \geqslant s\right\}$, putting $H_{t}:=\left\{s \in[0,1] \mid \mathbf{P}\left\{\zeta \in c\left(B_{d}, u, s\right)\right\} \leqslant t\right\}, t \in[0,1]$, for some $u \in \mathscr{S}_{d-1}$ (note that, by symmetry, this definition does not depend on the choice of $u$ ) and observing that $H_{t}$ has the form $[1-\widehat{H}(t), 1]$, we conclude that

$$
\Psi(t)=\Psi_{X, Y}(t)=\widehat{H}(t) .
$$

We will prove the following lemma characterising the behaviour of $\Psi(t)=\widehat{H}(t)$ in the neighbourhood of 0 .

Lemma 1. With the current notation $\Psi(0)=0$ and $\Psi$ is absolutely continuous in a neighbourhood of 0 with density

$$
\varphi(t)=\frac{a_{d}}{2 \Gamma(2 /(d+1))} t^{-(d-1) /(d+1)}+o\left(t^{-(d-1) /(d+1)}\right),
$$

where $a_{d}$ is given by (8).

Further, we check that $\Psi$ satisfies the condition (REG), which is easily seen to follow from the statement of the lemma given below.

Lemma 2. For $\Psi$ given by (10)

$$
\mathbf{E} \widehat{\Lambda}\left(\xi_{1}, \xi_{2}, \eta_{1}, \eta_{2}\right) 1_{\left\{\eta_{1} \in H_{t}\right\}} 1_{\left\{\eta_{2} \in H_{t}\right\}}=O\left(\Psi^{2}(t) t^{1+(d-1) /(d+1)}\right),
$$

where $\xi_{1}, \xi_{2}$ and $\eta_{1}, \eta_{2}$ are jointly independent copies of $\xi$ and $\eta$ respectively and

$$
\widehat{\Lambda}\left(u_{1}, u_{2}, s_{1}, s_{2}\right)=\mathbf{P}\left\{\zeta \in c\left(B_{d}, u_{1}, s_{1}\right) \cap c\left(B_{d}, u_{2}, s_{2}\right)\right\} .
$$

Lemmas 1 and 2 (to be proven in the next section) yield Theorem 6 as a direct consequence of Corollary 1 and Theorem 5 .

It is to be emphasised that the methods used in the proof of Theorem 6 can be applied, with only slight changes, to establish analogous results for $B_{d}$ replaced by a general convex set with sufficiently smooth boundary and for the common distribution of $\zeta_{1}, \zeta_{2}, \ldots$ which is regular enough, but not necessarily uniform. Further generalisation would be to consider, instead of the mean width given by $(7)$, the integral of $h\left(C_{n}, \cdot\right)$ with respect to a sufficiently regular nonuniform measure on $\mathscr{S}_{\boldsymbol{d - 1}}$, which could be of interest as providing the asymptotics of mixed volumes $V\left(C_{n}, K_{1}, \ldots, K_{d-1}\right)$ (see Chapter 5 in [16]) for convex $K_{1}, \ldots, K_{d-1}$ smooth enough. The detailed formulation of conditions under which these generalisations can be carried on is the subject of work in progress. In the present paper, for simplicity and brevity, we confine ourselves to the simplest case of the uniform distribution on a ball. 
3. Proofs. In the proofs of Theorems 1 and 3 we are going to use the following simple lemma.

Lemma 3. Let $\eta_{1}^{(i)}, \eta_{2}^{(i)}, \ldots$ for $i=1,2$ and $\beta_{1}, \beta_{2}, \ldots$ be certain sequences of real numbers. Then

1) if $\beta_{n} \rightarrow \infty$ then

$$
\limsup _{n \rightarrow \infty} \frac{\ln \left(\eta_{n}^{(1)}+\eta_{n}^{(2)}\right)}{\ln \beta_{n}} \leqslant \max _{i=1,2} \limsup _{n \rightarrow \infty} \frac{\ln \eta_{n}^{(i)}}{\ln \beta_{n}}
$$

2) if $\beta_{n} \rightarrow 0$ then

$$
\liminf _{n \rightarrow \infty} \frac{\ln \left(\eta_{n}^{(1)}+\eta_{n}^{(2)}\right)}{\ln \beta_{n}} \geqslant \max _{i=1,2} \liminf _{n \rightarrow \infty} \frac{\ln \eta_{n}^{(i)}}{\ln \beta_{n}} .
$$

P r o o f. We prove only the first part of the lemma (the proof of the second one being analogous). We have $\ln \left(\eta_{n}^{(1)}+\eta_{n}^{(2)}\right) / \ln \beta_{n} \leqslant \ln \left(2 \max _{i=1,2} \eta_{n}^{(i)}\right) /$ $\ln \beta_{n}=\ln 2 / \ln \beta_{n}+\max _{i=1,2}\left(\ln \eta_{n}^{(i)}\right) / \ln \beta_{n}$. Since $\beta_{n} \rightarrow \infty$ we get $\ln 2 / \ln \beta_{n} \rightarrow 0$. Taking the upper limits completes the proof.

3.1. Proof of Theorem 1. Since $Y$ and $X_{1}, X_{2}, \ldots$ are independent we have $\mathbf{P}\left\{X^{(n)} \cap Y=\varnothing\right\}=\int_{\mathscr{F}} \mathbf{P}\left\{X^{(n)} \cap F=\varnothing\right\} d \mathbf{P}^{Y}(F)=\int_{\mathscr{F}} \mathbf{P}\left\{X \dot{ }^{\dot{1}} F=\right.$ $\varnothing\}^{n} d \mathbf{P}^{Y}(F)$, where $\mathbf{P}^{Y}$ denotes the distribution of $Y$. By (2) and (3) this integral is equal to $\mathbf{E}\left(1-T_{X}(Y)\right)^{n}=\int_{[0,1]}(1-t)^{n} d \Psi(t)$. Hence, setting $\varepsilon_{n}=(\ln n)^{2} / n$, we get

$$
\frac{\ln \mathbf{P}\left\{X^{(n)} \cap Y=\varnothing\right\}}{\ln n}=\frac{\ln \int_{[0,1]}(1-t)^{n} d \Psi(t)}{\ln n} \leqslant \frac{\ln \left(\Psi\left(\varepsilon_{n}\right)+\left(1-\varepsilon_{n}\right)^{n}\right)}{\ln n} .
$$

Applying the first assertion of Lemma 3 for $\eta_{n}^{(1)}=\Psi\left(\varepsilon_{n}\right), \eta_{n}^{(2)}=\left(1-\varepsilon_{n}\right)^{n}$ and $\beta_{n}=n$ we obtain

$$
\begin{aligned}
& \limsup _{n \rightarrow \infty} \frac{\ln \mathbf{P}\left\{X^{(n)} \cap Y=\varnothing\right\}}{\ln n} \\
& \quad \leqslant \max \left(\limsup _{n \rightarrow \infty} \frac{\ln \Psi\left(\varepsilon_{n}\right)}{\ln n}, \limsup _{n \rightarrow \infty} \frac{n \ln \left(1-\varepsilon_{n}\right)}{\ln n}\right) .
\end{aligned}
$$

However, it is easily seen that $\lim _{n \rightarrow \infty} n \ln \left(1-\varepsilon_{n}\right) / \ln n=-\infty$. Further, $\lim _{n \rightarrow \infty} \ln \varepsilon_{n} / \ln n=-1$, so

$$
\limsup _{n \rightarrow \infty} \frac{\ln \Psi\left(\varepsilon_{n}\right)}{\ln n}=\limsup _{n \rightarrow \infty} \frac{\ln \Psi\left(\varepsilon_{n}\right)}{\ln \varepsilon_{n}} \frac{\ln \varepsilon_{n}}{\ln n}=-\liminf _{n \rightarrow \infty} \frac{\ln \Psi\left(\varepsilon_{n}\right)}{\ln \varepsilon_{n}} .
$$

These remarks combined with (12) yield the upper bound

$$
\limsup _{n \rightarrow \infty} \frac{\ln \mathbf{P}\left\{X^{(n)} \cap Y=\varnothing\right\}}{\ln n} \leqslant-\liminf _{t \rightarrow 0} \frac{\ln \Psi(t)}{\ln t} .
$$

To prove the lower bound observe that

$$
\begin{aligned}
\frac{\ln \mathbf{P}\left\{X^{(n)} \cap Y=\varnothing\right\}}{\ln n} & =\frac{\ln \int_{[0,1]}(1-t)^{n} d \Psi(t)}{\ln n} \geqslant \frac{\ln \left((1-1 / n)^{n} \Psi(1 / n)\right)}{\ln n} \\
& =-\frac{\ln \left((1-1 / n)^{n}\right)+\ln \Psi(1 / n)}{\ln (1 / n)} .
\end{aligned}
$$


Hence, we get immediately

$$
\liminf _{n \rightarrow \infty} \frac{\ln \mathbf{P}\left\{X^{(n)} \cap Y=\varnothing\right\}}{\ln n} \geqslant-\limsup _{t \rightarrow 0} \frac{\ln \Psi(t)}{\ln t} .
$$

Combining (13) and (14) completes the proof.

3.2. Proof of Theorem 2. Choose arbitrary $\eta>0$ and let $\delta$ be such that $c(1-\eta) t^{\alpha} \leqslant \varphi(t) \leqslant c(1+\eta) t^{\alpha}$ for $t \leqslant \delta$. We have

$$
\begin{aligned}
n^{\alpha+1} \mathbf{P}\left\{X^{(n)} \cap Y=\varnothing\right\} & =n^{\alpha+1} \int_{[0,1]}(1-t)^{n} d \Psi(t) \\
& =n^{\alpha+1} \int_{0}^{\delta}(1-t)^{n} \varphi(t) d t+n^{\alpha+1} \int_{(\delta, 1]}(1-t)^{n} d \Psi(t) .
\end{aligned}
$$

Obviously, the second term converges exponentially to 0 , while the first one lies between $c(1-\eta) n^{\alpha+1} \int_{0}^{\delta}(1-t)^{n} t^{\alpha} d t$ and $c(1+\eta) n^{\alpha+1} \int_{0}^{\delta}(1-$ $t)^{n} t^{\alpha} d t$. Since $\eta$ is arbitrary, the Theorem will be proven if we show that $\lim _{n \rightarrow \infty} n^{\alpha+1} \int_{0}^{\delta}(1-t)^{n} t^{\alpha} d t=\Gamma(\alpha+1)$. This, however, follows immediately from $n^{\alpha+1} \int_{0}^{\delta}(1-t)^{n} t^{\alpha} d t=\int_{0}^{n \delta}\left(1-\frac{t}{n}\right)^{n} t^{\alpha} d t$. The proof is complete.

3.3. Proof of Theorem 3. First we consider the case $0<\gamma<\infty$. The main step of the proof is to show that

$$
\lim _{t \rightarrow 0} \frac{\ln \Psi_{X, Y^{(k)}}(t)}{\ln \Psi^{* k}(t)}=1,
$$

where $\Psi_{X, Y^{(k)}}$ is the distribution function of the random variable $T_{X}\left(Y^{(k)}\right)$ (see (2)) and $\Psi^{* k}$ is the distribution function of $\sum_{i=1}^{k} T_{X}\left(Y_{i}\right)$. In other words, $\Psi^{* k}$ is the $k$-th convolution power of $\Psi$. Since $\Psi^{k}(t / k) \leqslant$ $\Psi^{* k}(t) \leqslant \Psi^{k}(t)$ and $\lim _{t \rightarrow 0} \ln \Psi(t / k) / \ln \Psi(t)=\lim _{t \rightarrow 0}(\ln \Psi(t / k) /(\ln (t / k)+$ $\ln k))(\ln t / \ln \Psi(t))=1$ for $0<\gamma<\infty$ (by (5)), we conclude that

$$
\lim _{t \rightarrow 0} \frac{\ln \Psi^{* k}(t)}{\ln \Psi(t)}=k \text {. }
$$

If we prove (15), then combining it with (16) will yield

$$
\lim _{t \rightarrow 0} \frac{\ln \Psi_{X, Y^{(k)}}(t)}{\ln t}=\lim _{t \rightarrow 0} \frac{\ln \Psi_{X, Y^{(k)}}(t)}{\ln \Psi^{* k}(t)} \frac{\ln \Psi^{* k}(t)}{\ln \Psi(t)} \frac{\ln \Psi(t)}{\ln t}=k \gamma .
$$

Then, applying Theorem 1 for $Y^{(k)}$ instead of $Y$ will complete the proof for $0<\gamma<\infty$.

It is not difficult to guess intuitively why (15) should hold. The idea is to show that, roughly speaking, if for some $F_{1}, \ldots, F_{k} \in \mathscr{F}$ the probability $\mathbf{P}\left\{X \cap \bigcup_{i=1}^{k} F_{i} \neq \varnothing\right\}=T_{X}\left(\bigcup_{i=1}^{k} F_{i}\right)$ is very small then the sets $F_{i}$ are usually very small too, so that $\mathbf{P}\left\{X \cap F_{i} \neq \varnothing \wedge X \cap F_{j} \neq \varnothing\right\}=\Lambda_{X}\left(F_{i}, F_{j}\right), i \neq j$ (see (6)) is negligible and, therefore, $T_{X}\left(\bigcup_{i=1}^{k} F_{i}\right)=\mathbf{P}\left\{X \cap \bigcup_{i=1}^{k} F_{i} \neq \varnothing\right\}$ is close to

$$
\sum_{i=1}^{k} \mathbf{P}\left\{X \cap F_{i} \neq \varnothing\right\}=\sum_{i=1}^{k} T_{X}\left(F_{i}\right)
$$


To prove (15) observe first that $\sum_{i=1}^{k} T_{X}\left(Y_{i}\right) \geqslant T_{X}\left(Y^{(k)}\right)$, so

$$
\Psi_{X, Y^{(k)}}(t)=\mathbf{P}\left\{T_{X}\left(Y^{(k)}\right) \leqslant t\right\} \geqslant \mathbf{P}\left\{\sum_{i=1}^{k} T_{X}\left(Y_{i}\right) \leqslant t\right\}=\Psi^{* k}(t) .
$$

Further, note that for $F_{1}, \ldots, F_{k} \in \mathscr{F}$

$$
\sum_{i=1}^{k} T_{X}\left(F_{i}\right)-T_{X}\left(\bigcup_{i=1}^{k} F_{i}\right) \leqslant \sum_{i<j \leqslant k} \Lambda_{X}\left(F_{i}, F_{j}\right) .
$$

Therefore, denoting $\theta_{i}(t):=\mathbf{1}_{\left\{T_{X}\left(Y_{i}\right) \leqslant t\right\}}$, we get

$$
\begin{aligned}
& \mathbf{E}\left(\left(\sum_{i=1}^{k} T_{X}\left(Y_{i}\right)-T_{X}\left(Y^{(k)}\right)\right) \prod_{i=1}^{k} \theta_{i}(t)\right) \leqslant \sum_{i<j \leqslant k} \mathbf{E}\left(\Lambda_{X}\left(Y_{i}, Y_{j}\right) \prod_{i=1}^{k} \theta_{i}(t)\right) \\
& =\left(\begin{array}{c}
k \\
2
\end{array}\right) \Psi(t)^{k-2} \mathbf{E}\left(\Lambda_{X}\left(Y_{1}, Y_{2}\right) \theta_{1}(t) \theta_{2}(t)\right) .
\end{aligned}
$$

The last equality follows from $\mathbf{E} \theta_{i}(t)=\Psi(t)$ in view of the independence of $Y_{i}$ 's (and, hence, of $\theta_{i}(t)$ 's). Taking into account that $\Lambda_{X}\left(Y_{1}, Y_{2}\right) \leqslant$ $t$ whenever $\theta_{1}(t) \theta_{2}(t) \neq 0$ we conclude that $\mathbf{E}\left(\Lambda_{X}\left(Y_{1}, Y_{2}\right) \theta_{1}(t) \theta_{2}(t)\right) \leqslant$ $t \mathbf{E}\left(\theta_{1}(t) \theta_{2}(t)\right)=t \Psi^{2}(t)$. This inequality together with (18) yields

$$
\mathbf{E}\left(\left(\sum_{i=1}^{k} T_{X}\left(Y_{i}\right)-T_{X}\left(Y^{(k)}\right)\right) \prod_{i=1}^{k} \theta_{i}(t)\right) \leqslant\left(\begin{array}{l}
k \\
2
\end{array}\right) t \Psi^{k}(t) .
$$

To proceed write

$$
\begin{aligned}
& \Psi_{X, Y^{(k)}}(t)=\mathbf{P}\left\{T_{X}\left(Y^{(k)}\right) \leqslant t\right\} \\
&= \mathbf{P}\left\{\left(T_{X}\left(Y^{(k)}\right) \leqslant t\right) \wedge\left(\sum_{i=1}^{k} T_{X}\left(Y_{i}\right)-T_{X}\left(Y^{(k)}\right)>t\right)\right\} \\
&+\mathbf{P}\left\{\left(T_{X}\left(Y^{(k)}\right) \leqslant t\right) \wedge\left(\sum_{i=1}^{k} T_{X}\left(Y_{i}\right)-T_{X}\left(Y^{(k)}\right) \leqslant t\right)\right\} \\
& \leqslant \mathbf{P}\left\{\left(T_{X}\left(Y^{(k)}\right) \leqslant t\right) \wedge\left(\sum_{i=1}^{k} T_{X}\left(Y_{i}\right)-T_{X}\left(Y^{(k)}\right)>t\right)\right\} \\
&+\mathbf{P}\left\{\sum_{i=1}^{k} T_{X}\left(Y_{i}\right) \leqslant 2 t\right\} .
\end{aligned}
$$

Since $T_{X}\left(Y_{i}\right) \leqslant t$ for $i=1, \ldots, k$ whenever $T_{X}\left(Y^{(k)}\right) \leqslant t$, we obtain by the standard Markov's inequality and (19)

$$
\begin{aligned}
& \mathbf{P}\left\{\left(T_{X}\left(Y^{(k)}\right) \leqslant t\right) \wedge\left(\sum_{i=1}^{k} T_{X}\left(Y_{i}\right)-T_{X}\left(Y^{(k)}\right)>t\right)\right\} \\
& \quad \leqslant \frac{\mathbf{E}\left(\left(\sum_{i=1}^{k} T_{X}\left(Y_{i}\right)-T_{X}\left(Y^{(k)}\right)\right) \prod_{i=1}^{k} \theta_{i}(t)\right)}{t} \leqslant\left(\begin{array}{c}
k \\
2
\end{array}\right) \Psi^{k}(t) .
\end{aligned}
$$


In view of $(20)$ it leads to

$$
\Psi_{X, Y^{(k)}}(t) \leqslant\left(\begin{array}{l}
k \\
2
\end{array}\right) \Psi^{k}(t)+\Psi^{* k}(2 t) .
$$

Note that since $\gamma>0$ relation (16) gives us $\lim _{t \rightarrow 0} \Psi^{* k}(t)=0$. Apply for each sequence $t_{n} \rightarrow 0$ the second assertion of Lemma 3 for $\eta_{n}^{(1)}=\left(\begin{array}{c}k \\ 2\end{array}\right) \Psi^{k}\left(t_{n}\right)$, $\eta_{n}^{(2)}=\Psi^{* k}\left(2 t_{n}\right)$ and $\beta_{n}=\Psi^{* k}\left(t_{n}\right)$ and observe that $\lim _{n \rightarrow \infty} \ln \eta_{n}^{(i)} / \ln \beta_{n}=1$, $i=1,2$ (for $i=1$ it follows from (16), for $i=2$ use (16) and (5)). Hence, (22) yields the lower bound $\lim \inf _{t \rightarrow 0} \ln \Psi_{X, Y^{(k)}}(t) / \ln \Psi^{* k}(t) \geqslant 1$. On the other hand, (17) gives us the upper bound $\lim \sup _{t \rightarrow 0} \ln \Psi_{X, Y^{(k)}}(t) /$ $\ln \Psi^{* k}(t) \leqslant 1$. Combining these bounds we obtain (15). This proves the Theorem for $0<\gamma<\infty$.

Assume now that $\gamma=0$. As in (17) we have $1 \geqslant \Psi_{X, Y^{(k)}}(t) \geqslant$ $\Psi^{* k}(t) \geqslant \Psi^{k}(t / k)$. Taking the logarithms and dividing both sides by $\ln t$ we get $0 \leqslant \ln \Psi_{X, Y^{(k)}}(t) / \ln t \leqslant k \ln \Psi(t / k) / \ln t$ and hence $\lim _{t \rightarrow 0} \ln \Psi_{X, Y^{(k)}}(t) /$ $\ln t=0$. Thus, our assertion for $\gamma=0$ follows from Theorem 1 applied for $Y^{(k)}$ instead of $Y$.

Further, let $\gamma=+\infty$. Then $0 \leqslant \Psi_{X, Y^{(k)}}(t) \leqslant \Psi(t)$. Therefore $+\infty \geqslant$ $\ln \Psi_{X, Y^{(k)}}(t) / \ln t \geqslant \ln \Psi(t) / \ln t$, so $\lim _{t \rightarrow 0} \Psi_{X, Y^{(k)}}(t) / \ln t=+\infty$. Applying once more Theorem 1 for $Y^{(k)}$ instead of $Y$ we get our assertion for $\gamma=+\infty$. The proof is complete.

3.4. Proof of Theorem 4. As in the previous Theorem, the main difficulty is to show that, roughly speaking, $\Psi_{X, Y^{(k)}}(t)$ is close to $\Psi^{* k}(t)$. Namely, we prove that

$$
\Psi_{X, Y(k)}(t)=\Psi^{* k}(t)+o\left(t^{k(\alpha+1)}\right) .
$$

Once this is established, it suffices to note that $\Psi^{* k}(0)=0$ and to show that $\Psi^{* k}$ is absolutely continuous in a neighbourhood of 0 with the density of the form

$$
\varphi^{* k}(t)=c^{k} \frac{\Gamma(\alpha+1)^{k}}{\Gamma(k(\alpha+1))} t^{k(\alpha+1)-1}+o\left(t^{k(\alpha+1)-1}\right)
$$

and, therefore,

$$
\Psi^{* k}(t)=c^{k} \frac{\Gamma(\alpha+1)^{k}}{\Gamma(k(\alpha+1)+1)} t^{k(\alpha+1)}+o\left(t^{k(\alpha+1)}\right) .
$$

These observations together with (23) give us the assertion of our theorem by arguments analogous to those used in the proof of Theorem 2 .

First we verify (24). Obviously

$$
\varphi^{* k}(t)=\int_{\left\{0 \leqslant t_{i}, t_{1}+t_{2}+\cdots+t_{k}=t\right\}} \varphi\left(t_{1}\right) \varphi\left(t_{2}\right) \cdots \varphi\left(t_{k}\right) d t_{1} d t_{2} \cdots d t_{k-1} .
$$


Since $\varphi(t)=c t^{\alpha}+o\left(t^{\alpha}\right),(24)$ results from the following equality, which is not difficult to establish

$$
\int_{\left\{0 \leqslant t_{i}, t_{1}+t_{2}+\cdots+t_{k}=t\right\}} t_{1}^{\alpha} t_{2}^{\alpha} \cdots t_{k}^{\alpha} d t_{1} d t_{2} \cdots d t_{k-1}=\frac{\Gamma(\alpha+1)^{k}}{\Gamma(k(\alpha+1))} t^{k(\alpha+1)-1} .
$$

Further, (25) is an immediate consequence of (24).

It remains to prove (23). To a great extent the proof of this relation goes along the same lines as the proof of (15) in Theorem 3. Indeed, taking into account that $\Psi(t)=O\left(t^{\alpha+1}\right)$, we conclude from (REG) and (18) that

$$
\mathbf{E}\left(\left(\sum_{i=1}^{k} T_{X}\left(Y_{i}\right)-T_{X}\left(Y^{(k)}\right)\right) \prod_{i=1}^{k} \theta_{i}(t)\right)=O\left(t^{k(\alpha+1)+1+\varepsilon}\right) .
$$

By analogy with (20)

$$
\begin{aligned}
& \Psi_{X, Y^{(k)}}(t)=\mathbf{P}\left\{T_{X}\left(Y^{(k)}\right) \leqslant t\right\} \\
& \leqslant \mathbf{P}\left\{\left(T_{X}\left(Y^{(k)}\right) \leqslant t\right) \wedge\left(\sum_{i=1}^{k} T_{X}\left(Y_{i}\right)-T_{X}\left(Y^{(k)}\right)>t^{1+\varepsilon / 2}\right)\right\} \\
& \quad+\mathbf{P}\left\{\sum_{i=1}^{k} T_{X}\left(Y_{i}\right) \leqslant t+t^{1+\varepsilon / 2}\right\} .
\end{aligned}
$$

As in (21), using Markov's inequality and (26) we get

$$
\begin{aligned}
& \mathbf{P}\left\{\left(T_{X}\left(Y^{(k)}\right) \leqslant t\right) \wedge\left(\sum_{i=1}^{k} T_{X}\left(Y_{i}\right)-T_{X}\left(Y^{(k)}\right)>t^{1+\varepsilon / 2}\right)\right\} \\
& \quad \leqslant \frac{\mathbf{E}\left(\left(\sum_{i=1}^{k} T_{X}\left(Y_{i}\right)-T_{X}\left(Y^{(k)}\right)\right) \prod_{i=1}^{k} \theta_{i}(t)\right)}{t^{1+\varepsilon / 2}}=O\left(t^{k(\alpha+1)+\varepsilon / 2}\right) .
\end{aligned}
$$

Together with (27) it leads to $\Psi_{X, Y^{(k)}}(t) \leqslant \Psi^{* k}\left(t+t^{1+\varepsilon / 2}\right)+O\left(t^{k(\alpha+1)+\varepsilon / 2}\right)$. By (25) it yields

$$
\Psi_{X, Y^{(k)}}(t) \leqslant \Psi^{* k}(t)+o\left(t^{k(\alpha+1)}\right) .
$$

Combining (17) and (28) we get (23). The proof is complete.

3.5. Proof of Lemma 1 . Denote by $G(s), s \in[0,1]$, the common value of $\mathbf{P}\left\{\zeta \in c\left(B_{d}, u, s\right)\right\}$ for $u \in \mathscr{S}_{d-1}$ (see the lines preceding (10) for the definition of $c\left(B_{d}, u, s\right)$ ). From (10) we conclude for $0 \leqslant s \leqslant 1$ that

$$
\Psi(G(1-s))=\widehat{H}(G(1-s))=s .
$$

In particular, $\Psi(0)=0$. On the other hand, we have the following useful relation (see (3.5) in [8] or [12])

$$
G(1-s)=\frac{\kappa_{d-1}}{\kappa_{d}} \int_{1-s}^{1}\left(1-t^{2}\right)^{(d-1) / 2} d t, \quad 0 \leqslant s \leqslant 1 .
$$


Thus,

$$
G(1-s)=2^{(d+1) / 2} \frac{\kappa_{d-1}}{(d+1) \kappa_{d}} s^{(d+1) / 2}(1+o(1)) .
$$

Hence (29) yields

$$
\Psi(t)=\frac{1}{2}\left(\frac{(d+1) \kappa_{d}}{\kappa_{d-1}}\right)^{2 /(d+1)} t^{2 /(d+1)}(1+o(1)) .
$$

To proceed observe that in view of $(29)$, since $G$ is invertible and its inverse is differentiable on $(G(1), G(0))=\left(0, \frac{1}{2}\right) ; \Psi$ is differentiable on $\left(0, \frac{1}{2}\right)$ too. Setting $g(t):=G^{\prime}(t), \varphi(t):=\Psi^{\prime}(t)$ and taking the derivatives in (29) we get $-\varphi(G(1-s)) g(1-s)=1$, or, substituting $t:=$ $G(1-s), \varphi(t)=-1 / g(1-\Psi(t))$. Together with $(30)$ this gives us $\varphi(t)=$ $\left(\kappa_{d} / \kappa_{d-1}\right)(\Psi(t)(2-\Psi(t)))^{-(d-1) / 2}$. Using (31) completes the proof.

3.6. Proof of Lemma 2. To find an upper bound for the expectation on the left-hand side of (11) observe first that $\widehat{\Lambda}\left(u_{1}, u_{2}, s_{1}, s_{2}\right)=0$ whenever $c\left(B_{d}, u_{1}, s_{1}\right) \cap c\left(B_{d}, u_{2}, s_{2}\right) \neq \varnothing$ and, moreover, $\widehat{\Lambda}\left(u_{1}, u_{2}, s_{1}, s_{2}\right)$ does not exceed $t$ provided $s_{1}, s_{2} \in H_{t}$. Thus, the left-hand side of (2) is bounded from above by $t P(t)$, where $P(t):=\mathbf{P}\left\{c\left(B_{d}, \xi_{1}, \eta_{1}\right) \cap c\left(B_{d}, \xi_{2}, \eta_{2}\right) \neq \varnothing\right.$, $\left.\eta_{1}, \eta_{2} \in H_{t}\right\}$. Since $\eta_{i}$ and $\xi_{i}$ are uniformly distributed (on $[0,1]$ and $\mathscr{S}_{d-1}$ respectively), $P(t)$ is proportional to the Lebesgue volume of the set of all the quadruples $\left(u_{1}, u_{2}, s_{1}, s_{2}\right) \in \mathscr{S}_{d-1}^{2} \times[0,1]^{2}$ satisfying

$$
\left.s_{i} \in H_{t} \text { (or, equivalently, } \operatorname{vol}\left(c\left(B_{d}, u_{i}, s_{i}\right)\right) \leqslant \kappa_{d} t\right), \quad i=1,2,
$$

and

$$
c\left(B_{d}, u_{1}, s_{1}\right) \cap c\left(B_{d}, u_{2}, s_{2}\right) \neq \varnothing .
$$

We will show that this volume is of order $O\left(\Psi^{2}(t) t^{(d-1) /(d+1)}\right)$, which will complete the proof. Note that once $\left(u_{1}, s_{1}\right)$ is chosen and (33) is to be fulfilled, the distance between $u_{1}$ and $u_{2}$ cannot exceed $\operatorname{diam}\left(c\left(B_{d}, u_{1}, s_{1}\right)\right)+$ $\operatorname{diam}\left(c\left(B_{d}, u_{2}, s_{2}\right)\right)$ for otherwise $c\left(B_{d}, u_{1}, s_{1}\right)$ and $c\left(B_{d}, u_{2}, s_{2}\right)$ would be disjoint. In view of (32) standard geometrical considerations yield $\operatorname{diam}\left(c\left(B_{d}, u_{i}, s_{i}\right)\right)=O\left(t^{1 /(d+1)}\right)$. This means that the surface measure of the part of the unit sphere $\mathscr{S}_{\boldsymbol{d}-1}$, where $u_{2}$ can lie is of order $O\left(t^{(\boldsymbol{d}-1) /(d+1)}\right)$. Hence, taking into account that (32) implies $s_{2} \in[1-\widehat{H}(t), 1]$, we conclude that the volume of the set of admissible $\left(u_{2}, s_{2}\right)$ given $\left(u_{1}, s_{1}\right)$ is of order $O\left(\widehat{H}(t) t^{(d-1) /(d+1)}\right)=O\left(\Psi(t) t^{(d-1) /(d+1)}\right)$ (see (10) and the preceding discussion). On the other hand, the volume of the set of pairs $\left(u_{1}, s_{1}\right)$ which do not violate (32) is proportional to $\Psi(t)$ (see ibidem). Hence

$$
P(t)=O\left(\Psi^{2}(t) t^{(d-1) /(d+1)}\right)
$$

as required. 
Acknowledgments. The author would like to express his gratitude to Prof. A. V. Nagaev for many valuable suggestions and comments. Special thanks are also due to the anonymous referee whose remarks have been very helpful in improving this paper.

\section{REFERENCES}

1. Bräker H., Hsing T. On the area and perimeter of a random convex hull in a bounded convex set. - Probab. Theory Relat. Fields, 1998, v. 111, № 4, p. 517-550.

2. Cabo A. J., Groeneboom P. Limit theorems for functionals of convex hulls. - Probab. Theory Relat. Fields, 1994, v. 100, №1, p. 31-55.

3. Efron B. The convex hull of a random set of points. - Biometrika, 1965, v. 52, p. 331-343.

4. Groeneboom P. Limit theorems for convex hulls. - Probab. Theory Relat. Fields, 1988 , v. 79 , № 3 , p. 327-368.

5. Hsing T. On the asymptotic distribution of the area outside a random convex hull in a disk. - Ann. Appl. Probab., 1994, v. 4, № 2, p. 478-493.

6. Kendall W.S., Mecke J., Stoyan D. Stochastic Geometry and Its Applications. Berlin: Akademie-Verlag, 1987, 345 p.

7. Хамдамов И. М., Нагаев А.В. Предельные распределения функционалов выпуклой оболочки, порожденной равномерно распределенными величинами. Докл. АН УзССР, 1991, т. 7, с. 8-9.

8. Küfer K. H. On the approximation of a ball by random polytopes. - Adv. Appl. Probab., 1994, v. 26, № 4, p. 876-892.

9. Matheron G. Random Sets and Integral Geometry. New York: Wiley, 1975, $261 \mathrm{p}$.

10. Molchanov I.S. Limit Theorems for Unions of Random Closed Sets. BerlinHeidelberg: Springer-Verlag, 1993, 157 p. (Lecture Notes in Math., v. 1561.)

11. Молчанов И. С. О сходимости случайных процессов, порожденных полиэдральными аппроксимациями выпуклых компактов. - Теория вероятн. и ее примен., 1995 , т. 40 , в. 2 , с. $438-444$.

12. Raynaud $H$. Sur le comportement asymptotique de l'enveloppe convexe d'un nuage des points tirés au hazard dans $\mathbf{R}^{n}$. - J. Appl. Probab., 1970, v. 7, p. 35-48.

13. Rényi A., Sulanke $R$. Über die konvexe Hülle von $n$ zufällig gewählten Punkten. I, II. - Z. Wahrscheinlichkeitstheor. verw. Geb., 1963, v. 2, p. 75-84; 1964, v. 3, p. $138-147$.

14. Resnick S.I. Extreme Values, Regular Variation, and Point Processes. BerlinHeidelberg: Springer-Verlag, 1987, 320 p.

15. Schneider R. Random approximation of convex sets. - J. Microscopy, 1988, v. 151, p. 211-227.

16. Schneider R. Convex bodies: the Brunn-Minkowski theory. Cambridge: Cambridge Univ. Press, 1993, 490 p. 